BNL $-\cdots 4803$

LE92 019248

\title{
Scaling of Gain with Energy Spread and Energy in the PEP FEL
}

\author{
A. S. Fisher
}

July 13,1992

Brookhaven National Laboratory Lpton. NY 11973

\section{DISCLAIMER}

\begin{abstract}
This report was prepared as an account of work sponsored by an agency of the United States Government. Neither the United States Givverrment nor any agency thereof, nor any' of their ernployees, makes any warranty. express or implied, or assumes any legal liability or responsibility for the accuracy, completeness, or usefalness of any information, apparatus, product, of process disclosed, or represents that its use would not infringe privately owned rights. Refer. ence herein to any' specific commercial product, process, or service by trade name, trademark, manufacturer. or otherwise does not necessarily constitute or imply is endorsement, recommendation, of favoring by the United States Government or any agency thereof. The views and opinions of authors expressad herein do not nocessurily state of reflect those of the United States Government or any agency thereof.
\end{abstract}

\section{MASTER}


BNL-47803

C.AP 85-92R

Informal Report

\title{
Scaling of Gain with Energy Spread and Energy in the PEP FEL
}

\author{
A. S. Fisher
}

July 13, 1992

Physics Department

Brookhaven National Laboratory

Associated Universitites, Inc.

Upton, Long Island, New York 11973

Under Contract No. DE-AC02-76CH00016 with the UNITED STATES DEPARTMENT OF ENERGY 


\section{Scaling of Gain with Energy Spread and Energy in the PEP FEL}

The Sag Harbor paper on the PEP FEL ${ }^{1}$ discusses the scaling of varions FEL param. eters with energy spread $\sigma_{\epsilon}$. I will repeat some of this material here and then examine the benefit of increasing the energy spread. How much energy spread can be achieved with damping wigglers is the next topic. Finally, I consider the dependence of gain and saturation length on beam energy and undulator field.

This note was originally written during a visit to SSRL in August 1991, and has been slightly modified since then.

\section{Review of the Scaling Rules}

The fractional rms energy spread $\sigma_{\epsilon}$ in a storage ring, determined by synchrotronradiation losses in the bending magnets, is proportional to beam energy and so favors low energy for the FEL. With low-ernittance optics in PEP (but without wigglers), "

$$
\sigma_{\epsilon 0}=6.6 \cdot 10^{-5} \cdot E[\mathrm{GeV}\}
$$

giving an energy spread of $2 \times 10^{-4}$ at $E=3 \mathrm{GeV}$.

Synchcotron radiation from a wiggler increases the beam's energy spread and thanges its emittance. Damping wigglers, which are placed in low or zero dispersion locations, increase energy spread but reduce emittance:

$$
\begin{aligned}
& \frac{\sigma_{\epsilon w}^{2}}{\sigma_{t(1)}^{2}}=\frac{1+\frac{\sqrt{2} \pi^{2} N_{w} R_{0}^{2} K_{w}^{3}}{\lambda_{w 0}^{2} \gamma^{3}}}{1+\frac{\pi N_{w} R_{0} K_{w}^{2}}{\lambda_{w} \gamma^{3}}}=\frac{1+P Q^{3}}{1+P Q^{2}}=R, \\
& \frac{\epsilon_{x w}}{\epsilon_{x(1)}}=\frac{1}{1+\frac{\pi N_{w} R_{0} K_{w}^{2}}{\lambda_{w} \gamma^{2}}}=\frac{1}{1+P Q^{2}} .
\end{aligned}
$$

(A small term in $\mathrm{Eq}_{1}$. (3) from dispersion in the wiggler has been dropped.) Herc

$$
K_{w}=\frac{e B_{w} \lambda_{w}}{2 \pi m_{e} c}=0.9337 B_{w}|\mathrm{~T}| \lambda_{w}|\mathrm{~cm}|
$$

is the dimensionless wiggler paraneter. expressed in terms of the wiggler period $\lambda_{u}$, and the peak wiggler field $B_{w}$ on axis; $N_{w}=L_{u} / \lambda_{w}$ is the number of periuds in a wiggler of length $L_{w} ; \gamma$ is the ratio of the total energy to rest energy of an clectron: and $R_{n}$. The

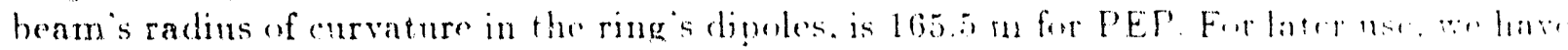
defined parameters $P$ and $Q$ :

$$
\begin{aligned}
& P=\frac{L_{u}}{2 \pi R_{0}} \quad \frac{L_{u} \mathrm{~m}}{10+1)} \\
& Q=\frac{\sqrt{2 \pi R_{1} K_{u}}}{\gamma \lambda_{u}}=35 \frac{B_{u}|\mathrm{~T}|}{E|\mathrm{GeV}|}
\end{aligned}
$$


Note that the wiggler period does not enter into the scaling, and so long periods with high fields can be used. $R$ is the square of the increase in energy spread.

For sufficiently long damping wigglers, the change in energy spread saturates at

$$
\frac{\sigma_{\epsilon w}^{2}}{\sigma_{\epsilon 0}^{2}}=\frac{\sqrt{2} \pi R_{0} K_{w}}{\lambda_{w} \gamma}=Q
$$

but the emittance will continue to decrease with $N_{w}$ (until the effect saturates due to the neglected dispersive term in Eq. (3).)

If several damping wigglers are distributed around the ring, then the effect is cumulative over their total length. However, an FEL wiggler in a bypass, with the beam switched in intermittently, will not contribute to the bearn's equilibriun energy spread and emittance.

A careful choice of a damping wiggler offers two benefits for the FEL. Low values of emittance are needed to lase at short wavelengths. At low energies, the ring's peak current is seriously limited; increasing the energy spread allows higher current.

The limit on the peak current $\hat{I}$ is imposed by the longitudinal microwave instability. For an estimate, we rely on the extrapolation of bunch-length measurements made on the SPEAR ring and scaled to fit PEP data. ${ }^{4-5}$ These scaling rules are modeled by the ZAP code ${ }^{6}$ which incorporates the following formula: ${ }^{7}$

$$
I \leq \frac{2 \pi \alpha \beta E \sigma_{\epsilon}^{2}}{\left|Z_{\|} / n\right| e}
$$

Here, $\alpha$ is the ring's momentum-compaction factor, the ratio of the fractional increase in the orbit path length to the fractional change in momentum; $\beta=v / c$ for an electron of energy $E_{;} e$ is the unit charge; $n=\omega / \omega_{0}$ is the mode number; and $\omega_{0}$ is the rerolution frequency in the ring. This can often be simplified using Eq. (1) (but not including the effect of damping wigglers (2)):

$$
\dot{I}_{\max } \propto E \sigma_{\varepsilon}^{2} \propto E^{3},
$$

The frequency of interest $\omega$ is not critical in most cases, since the imperlance is inductive. leaving $\left|Z_{\|} / n\right|$ constant. Measurements made at $4.5 \mathrm{GeV}{ }^{8}$ with rms bunch lengths $\sigma$, of several centimeters, agree with these extrapolations. However. for frequencies above $\omega_{r}=c / b$, where $b$ is the beam-pipe radius, $\left|Z_{\|} / n\right|$ decreases. SPEAR scaling gives $\left|Z_{\| / /}\right| x$ $\left(\omega / \omega_{r}\right)^{1.68}$, and ZAP uses $\omega=c / \sigma_{s}$. For extremely short bunches. ZAP does not allow the impedance to decrease below the "free-space" longitudinal impedance of the bending magnets. screened by the beam-pipe wall: $\left|Z_{||} / n\right|=300\left(b / R_{n v}\right)$, where $R_{\mathrm{nv}}=L_{R /} /(2 \pi)$ is the average radius of a ring of circumference $L_{R}$. In our case, with its very short bunches. ZAP uses this free-space limit: for PEP's lowemittance mode and an cherke if a (3). ZAP gives a maximum peak current of $17.6 \mathrm{~A}$.

In the exponential gain regime of an FEL with undulator parameters $k_{\|}$and $\lambda_{1}$. the power grows with an e-folding length

$$
L_{G}=\frac{\lambda_{u}}{4 \pi \sqrt{3} \rho} .
$$


The dimensionless gain parameter $\rho$ is given by:

$$
\frac{1}{L_{u}^{3}}=\frac{\rho^{3}}{\lambda_{u}^{3}}=\frac{r_{e}}{64 \pi^{2} e c} \frac{K_{u}{ }^{2}}{\lambda_{u} \sigma_{x} \sigma_{y}} \frac{\hat{I}}{\gamma^{3}}\left(J_{0}-J_{1}\right)^{2} .
$$

Here $L_{u}$ is the necessary undulator length for saturation:

$$
L_{u}=\lambda_{u} / \rho=4 \pi \sqrt{3} L_{G}=21.8 L_{G} .
$$

$J_{0}$ and $J_{1}$ are Bessel functions with the argument

$$
\xi=\frac{K_{u}^{2} / 4}{1+K_{u}^{2} / 2}
$$

Since the argument takes on values from 0 to 0.5 , the squared difference of Bessel functions ranges from 1 to 0.485 .

When the energy spread $\sigma_{\epsilon}$ approaches $\rho$, the gain is reduced: ${ }^{9}$

$$
\frac{\rho_{\mathrm{eff}}}{\rho}=\frac{\exp \left[-0.136\left(\sigma_{\epsilon} / \rho\right)^{2}\right]}{1+0.64\left(\sigma_{\epsilon} / \rho\right)^{2}} .
$$

The increase of $\sigma_{\epsilon}$ with energy (1) and damping wigglers (2) argue for a low energy to avoid this gain reduction. This topic will be expored in Section 4 .

The beam sizes $\sigma_{x}$ and $\sigma_{y}$ are limited both by a restriction on focusing,

$$
\beta_{y} \geq \beta_{x} \geq L_{G}
$$

and by diffraction, which is expressed in terms of the Rayleigh length of the $\mathrm{x}$ rays:

$$
z_{R}=\pi w_{0}^{2} / \lambda=4 \pi \sigma_{y}^{2} / \lambda \geq L_{G} .
$$

(The Rayleigh length is defined in terms of the $1 /$ e radius $w_{0}$ of the electric field. Here we equate $\sigma_{y}$, the smaller of the two rms electron-beam radii, not to $w_{0}$ but to the rms radius of the $x$-ray power.) This diffractive limit proves to be cquite severe and prevents our focusing to a $\sigma_{y}$ smaller than $\sigma_{x}$. Hence we set

$$
\sigma_{x}=\sigma_{y}
$$

\section{Optimization of the Energy Spread}

We see from Eq. (9) that a higher energy spread will increase the peak surrent. and hence $\rho$, but will lead to a greater gain reduction from $E_{(1 .}(14)$. Where is the optimum? For a given energy, we have

$$
\rho^{3} \propto I \propto \sigma_{\epsilon}^{2} .
$$


Use this scaling to define parameters $S, T$, and $U$ that are independent of $\sigma_{\varepsilon}$ and $\rho$ :

$$
\begin{aligned}
\frac{\sigma_{\epsilon}{ }^{2}}{\rho^{2}} & =S \sigma_{\epsilon}{ }^{2 / 3}, \\
\rho & =T S \sigma_{\epsilon}{ }^{2 / 3}, \\
I & =U \sigma_{\epsilon}{ }^{2} .
\end{aligned}
$$

Then for $\rho_{\text {eff }}$ we have:

$$
\rho_{\text {eff }}=T\left(S \sigma_{\epsilon}{ }^{2 / 3}\right) \frac{\exp \left[-0.136\left(S \sigma_{\epsilon}{ }^{2 / 3}\right)\right]}{1+0.64\left(S \sigma_{\epsilon}{ }^{2 / 3}\right)} .
$$

Eq. (22) has a peak, but to find it we need values for $S, T$, and $U$ for a particular choice of beam and undulator parameters. Start with the values on line 2 of the Sag Harbor ${ }^{1}$ or $\mathrm{PAC} 91^{10}$ table (the case with a $3.5-\mathrm{GeV}$ energy and a hybrid magnet with a 1-cm gap):

$$
\begin{aligned}
\sigma_{\epsilon} & =7.2 \times 10^{-4}, \\
\rho & =9.1 \times 10^{-4}, \\
I & =219 \mathrm{~A} .
\end{aligned}
$$

These give

$$
\begin{aligned}
& S=78, \\
& T=1.45 \times 10^{-3}, \\
& U=4.22 \times 10^{8} \mathrm{~A} .
\end{aligned}
$$

The conserquences of these scaling rules are shown in Table 1. Neglect for the moment the unrealistic values near the bottom of the table. Because of the strong scaling of peak current, the peak of $\rho_{\text {eft }}$ is $1.0 \times 10^{-3}$, for a $\sigma_{\epsilon}$ of $6 \times 10^{-3}$. In comparison, the Sag Harbor result, which is also repeated in this table, uses the more cautious energy spread of $7.2 \times 10^{-4}$ to get a $\rho_{\text {eff }}$ of $6.0 \times 10^{-4}$.

Varions effects will limit the range of these scaling rules. A damping wiggler is limited by the saturation value ( 7 ) in how much energy spread it can introduce: this limit will be considered in the next section. A higher beam energy raises the energy spread achievable with a damping wiggler and raises the peak current; this is discussed in Section 4 . PEP's rf acceptance will restrict $\sigma_{\epsilon}$ to perhaps $2 \times 10^{-3}$, but, even at this value, a large increase in gain can be achieved. I am not certain which effect will linit $I$. but it conll perhaps m." to 1 or $2 \mathrm{kA}$. (The linit on average current is considered in the next section.) Ans increnst. obtained in $\rho_{\text {eff }}$ corresponds to a proportionate decrease in $L_{G}$ and $L_{w}$. In addition. the shorter gain length allows for tighter focusing (a shorter $\beta_{x}$ and a shorter Rayleigh length and the possibility of a further increase in gain. 


\begin{tabular}{|c|c|c|c|}
\hline $\begin{array}{c}\sigma_{\epsilon} \\
\left(10^{-4}\right)\end{array}$ & $\begin{array}{c}\hat{I} \\
(\mathrm{~A})\end{array}$ & $\begin{array}{c}\rho \\
\left(10^{-4}\right)\end{array}$ & $\begin{array}{c}\rho_{\text {eff }} \\
\left(10^{-4}\right)\end{array}$ \\
\hline 2 & 17 & 3.9 & 3.2 \\
4 & 68 & 6.1 & 4.6 \\
6 & 152 & 8.1 & 5.5 \\
7.2 & 219 & 9.1 & 6.0 \\
8 & 270 & 9.8 & 6.2 \\
9.2 & 361 & 10.7 & 6.6 \\
10 & 422 & 11.3 & 6.8 \\
12 & 608 & 12.8 & 7.3 \\
17 & 1220 & 16.1 & 8.1 \\
20 & 1690 & 18.0 & 8.5 \\
30 & 3800 & 23.6 & 9.3 \\
40 & 6760 & 28.5 & 9.7 \\
50 & 10600 & 33.1 & 9.9 \\
60 & 15200 & 37.4 & 10.0 \\
80 & 27000 & 45.3 & 9.9 \\
100 & 42200 & 52.6 & 9.7 \\
200 & 169000 & 83.5 & 8.2 \\
\hline
\end{tabular}

Table 1: Effect of energy spread on gain, using Eq. (22).

\section{Optimization of the Damping Wiggler}

Table 1 suggests that the highest possible energy spread is desirable. A damping wiggler is limited by $\mathrm{Eq}$. (7) in the increase in $\sigma_{\epsilon w}$ it can provide. Since this limit $Q$ depends only on the wiggler field (for the $3.5-\mathrm{GeV}$ energy considered here), we choose the largest field conveniently achievable. For a room-temperature magnet:

$$
\begin{aligned}
B_{w} & =2 \mathrm{~T} \\
Q & =20.0 .
\end{aligned}
$$

Next, we choose a large value of the energy-spread increase factor $R$ (limited by $Q$ ), and solve Eq. (2) for $P$ (the wiggler length):

$$
P=\frac{R-1}{Q^{2}(Q-R)}
$$

Since $P>0$. we see that $Q>R>1$, and $R$ cannot get tou close to $Q$ wr the wiggler length will diverge. A reasonable choice might be:

$$
\begin{aligned}
R & =16 . \\
P & =9.38 \times 10^{3} . \\
\sigma_{\text {rw }} & =9.2 \times 10^{-4}, \\
L_{w} & =9.7 \mathrm{~m} .
\end{aligned}
$$


The wiggler period is not specified, and can be chosen for convenience in achieving the $2-T$ field. The current and $\rho$ values corresponding to this choice are listed in Table 1 . We see that this choice of damping wiggler raises $\rho_{\text {eff }}$ from $6.0 \times 10^{-4}$ to $6.6 \times 10^{-4}$.

A higher damping-wiggler field would give a greater energy spread and a higher peak current. For a superconducting wiggler:

$$
\begin{aligned}
B_{w} & =6 \mathrm{~T}, \\
Q & =60.0 .
\end{aligned}
$$

Again, we choose a value of $R$ near $Q$ and solve for $P$ :

$$
\begin{aligned}
R & =56, \\
P & =3.82 \times 10^{-3}, \\
\sigma_{\epsilon w} & =1.7 \times 10^{-3}, \\
L_{w} & =4.0 \mathrm{~m} .
\end{aligned}
$$

Table 1 shows that $\rho_{\text {eff }}$ for this choice is $8.1 \times 10^{-4}$.

In reality, this energy spread may be a bit too large for PEP's rf acceptance. Also, the single-bunch current limit may be exceeded in this case. To check this, we need to find the average current. The bunch length in the Sag Harbor table is $4.2 \mathrm{~mm}$, and this length is proportional to energy spread. We get:

$$
\begin{aligned}
\sigma_{z} & =9.9 \mathrm{~mm}, \\
I_{\mathrm{av}} & =\sqrt{2 \pi} \hat{I} \sigma_{z} / L_{R}=13.8 \mathrm{~mA} .
\end{aligned}
$$

( $L_{R}$ is the ring circumference.) Heinz-Dieter Nuhn (SSRL) was not certain, but he suggested that the limit is $10 \mathrm{~mA}$.

\section{Optimization of the Energy}

Combine Eqs. (1) and ( 7 ) to find the dependence of energy spread (with a damping wiggler) on $\gamma$ :

$$
\sigma_{\epsilon w}^{2} \propto \gamma^{2} Q \propto \gamma
$$

( $B_{w}$ is held fixed at $2 \mathrm{~T}$, following Eq. (29).) Using Eq. (9), the peak current's dependence then is:

$$
\hat{I} \propto \gamma^{2} .
$$

We want to maintain a fixed wavelength,

$$
\lambda=\lambda_{u} \frac{1+K_{u}^{2} / 2}{2 \gamma^{2}}
$$

while maximizing the gain or minimizing the undulator length. both using E(1. (11). The' beam size scaling, which comes either from Eql. (15) or $E_{1}$. (16), is the same for either limit (since a damping wiggler can provide a sufficiently low emittance to allow ; to scalo freely):

$$
\sigma_{x}^{2}=\sigma_{y}^{2} \propto L_{G} \times \lambda_{u} / \rho
$$




\section{(a) High-Field Undulators}

The premise in designing the high field FELs was minimizing the undulator saturation length $L_{u}$ in Eq. (11). Substitute for $\hat{I}$ from Eq. (45), for $\lambda_{u}$ from Eq. (46) (with constant wavelength), and for $\sigma_{x} \sigma_{y}$ from $\mathrm{Eq} .(47)$ :

$$
\frac{1}{L_{u}^{2}}=\frac{\prime^{2}}{\lambda_{u}^{2}} \propto \frac{K_{u}^{2}\left(1+K_{u}^{2} / 2\right)}{\gamma^{3}} .
$$

We need a li rge $K_{u}$ and a small $\gamma$.

First we regard the magnetic field as an independent parameter, because, for example, a variety of undulator designs are being compared, or because any one design need not be pushed to its high-field limit. To maintain synchronisin, $\lambda_{u}$ must decrease even though $K_{u}$ is increasing. For large $K_{u}$ and constant wavelength, Eq. (46) gives:

$$
\lambda_{u} \propto\left(\frac{\gamma}{B_{u}}\right)^{2 / 3},
$$

and the saturation length scaling becomes:

$$
L_{u} \propto \gamma^{1 / 6} / B_{u}^{2 / 3} .
$$

As a result, we choose a low energy and the largest practical field, limited by the properties of materials such as samarium cobalt. Then, as $\lambda_{u}$ gets smaller, the gap must be reduced to maintain the field on axis, until the limit of an unacceptable gap is reached.

Now consider operation at the high-field limit of any one design, with the minimum gap acceptable for injection and beam lifetime (especially if the undulator is in the main ring rather than a bypass). The magnetic field now is linked to the undulator period and, through Eq. (46), to the beam energy. For example, the field of a hybrid, planar wiggler using neodymium-iron permanent magnets is given by

$$
B_{w}[\mathrm{~T}]=3.44 \exp \left[-\left(g / \lambda_{w}\right)\left(5.08-1.54 g / \lambda_{w}\right)\right] .
$$

Such expressions do not lead to any simple scaling, but it may be seen that there is an optimum energy and period giving the shortest saturation length. This value must be found iteratively: at each of a range of energies, find $\lambda_{u}$ and the corresponding maximum $B_{u}$ that give the desired wavelength, and calculate the saturation length.

\section{(b) Cusp-Field Undulators}

Roman Tatchyn's premise in this case ${ }^{10}$ is maximizing $\rho$ while holding $I$ fixed. The overall undulator length is not as important here, because the cusp-field undulator is simply and sparsely constructed, and can have excellent field accuracy over long lengths. Making the same substitutions hefore, we get:

$$
\rho^{2} \propto\left(\frac{K_{u}^{2}}{1+K_{u}^{2} / 2}\right) \gamma
$$


Again, we need a large $K_{u}$, although this time the improvement saturates for $K_{u}$ values much above 3 . In contrast to Eq. (50), the energy should be large, while $\lambda_{u}$ can increase to maintain synchronism following $\mathrm{Eq}_{\mathrm{q}}$. (49). The demand on field strength is not as great. The limit to this procedure arrives when the undulator length fills PEP's straight section ( $\approx 90 \mathrm{~m}$, to allow for matching the short beta function of the FEL).

\section{References}

1. A.S. Fisher, S. Baird, H.-D. Nuhn, R. 'Tatchyn, H. Winick and C. Pellegrini, "A $40-\AA$ FEL on the PEP Storage Ring," Proc. Workshop on Prospects for a $1-\AA$ FreeElectron Laser, Sag Harbor, NY, 22-27 April 1990, J.C. Gallardo, ed., BNL 52273, p. 41.

2. A. Hofmann, "Short Bunches in PEP," SSRL ACD Note 39, Nov. 1986.

3. H. Wiedemann, Nucl. Instrum. Methods A268, 24 (1988).

4. A.W. Chao and J. Gareyte, "Scaling Law for Bunch Lengthening in SPEAR II," SPEAR 197, PEP 224 (1976).

5. M. Month, "Bunch Lengthening in SPEAR and Extrapolation to PEP," SPEAR 198, PEP 227 (1976).

6. M.S. Zisman, S. Chattopadhyay, and J.J. Bisognano, "ZAP User's Manual," LBL $21270,1986$.

7. A. Hofmann, "Sirgle Beam Collective Phenomena-Longitudinal," Proc. $1^{\text {st }}$ International School of Particle Accelerators, Erice 1976, CERN 77-13, 139 (1977).

8. M. Donald, R. Helm, A. Hofmann, 'T. Martin, P. Morton, S. Myers, J.M. Paterson, J.-L. Pellegrin, L. Rivicin, R. Ruth, and P. Wilson, "Feedback Experiment at PEP," LEP Note 553 (1986).

9. G. Dattoli, A. Renieri, A. Torre, and R. Caloi, Nuovo Cimento 11D, 393 (1989).

10. S. Baird, H.-D. Nuhn, R. Tatchyn, H. Winick, A.S. Fisher, J.C. Gallardo, C. Pellegrini, "Coherent X-Rays from PEP," Proc. IEEE Particle Accelerator Conf., San Francisco, CA, 6.9 May 1991 (IEEE. Piscataway, NJ), p. 2748. 

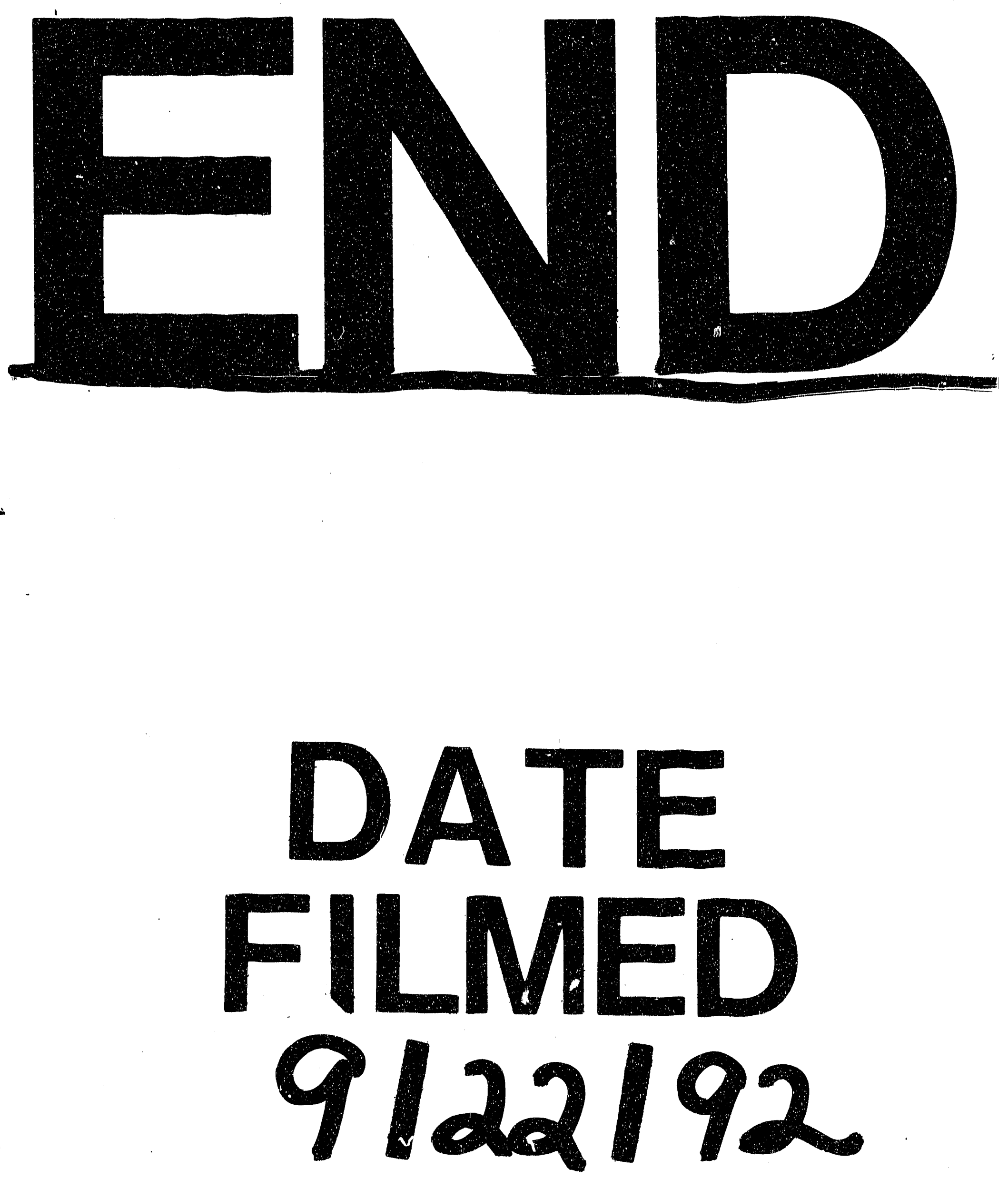
